“ (C) 2017 IEEE. Personal use of this material is permitted. Permission from IEEE must be obtained for all other uses, in any current or future media, including

reprinting/republishing this material for advertising or promotional purposes, creating new collective works, for resale or redistribution to servers or lists, or reuse of any copyrighted component of this work in other works." 


\title{
EEG-based Emotion Classification Using Innovative Features and Combined SVM and HMM Classifier
}

\author{
Kairui Guo, Henry Candra, Hairong Yu,Huiqi Li, Hung T. Nguyen and Steven W. Su
}

\begin{abstract}
Emotion classification is one of the state-of-theart topics in biomedical signal research, and yet a significant portion remains unknown. This paper offers a novel approach with a combined classifier to recognise human emotion states based on electroencephalogram (EEG) signal. The objective is to achieve high accuracy using the combined classifier designed, which categorises the extracted features calculated from time domain features and Discrete Wavelet Transform (DWT). Two innovative designs are involved in this project: a novel variable is established as a new feature and a combined SVM and HMM classifier is developed. The result shows that the joined features raise the accuracy by $5 \%$ on valence axis and $1.5 \%$ on arousal axis. The combined classifier can improve the accuracy by 3\% comparing with SVM classifier. One of the important applications for high accuracy emotion classification system is offering a powerful tool for psychologists to diagnose emotion related mental diseases and the system developed in this project has the potential to serve such purpose.
\end{abstract}

\section{INTRODUCTION}

Emotion classification has been brought as a diagnosing tool by psychologists for more than 200 years. From psychological point-of-view, emotions belong to the second, socalled affective sphere of mental functioning, which includes the emotions themselves, moods, evaluations, and other feeling states, including fatigue or energy [1]. According to psychologists' observation and experience, decisions on the patients' emotion states are made based on facial expressions and gestures. Nevertheless, this method has a major flaw that the result of the classification is entirely empirical, and the accuracy of this approach is not able to be calculated quantitatively. Moreover, issues related to advanced humanmachine interaction, brain-computer interface [2] and medical care [3] also require a stable emotion classification system. Thus, a more accurate and dependable classification system is demanded. In the last few decades, biomedical engineers have expanded their research fields into emotion classification. Anatomically, human emotions are generated from the limbic system that is located in the frontal lobe of the brain. To accurately classify emotions, it is preferable to analyse signal from the limbic system. Therefore, electroencephalogram (EEG), which represents the electrical activities of the brain, shall be chosen in emotion classification study.

Kairui Guo, Henry Candra, Hung T. Nguyen and Steven Su are with the Centre for Health Technologies, Faculty of Engineering and Information Technology, University of Technology, Sydney, New South Wales, Australia (email: kairui.guo@student.uts.edu.au, henry.candra@student.uts.edu.au, hairong.yu@student.uts.edu.au, hung.nguyen@uts.edu.au, steven.su@uts.edu.au). Huiqi Li is with the School of Information and Electronics, Beijing Institute of Technology (email:huiqili@bit.edu.cn).
In this paper, a high efficiency and stable emotion classification system is designed based on EEG signal using wavelet analysis in feature extraction and a novel approach of combined Support Vector Machine (SVM) and Hidden Markov Model (HMM) classifier in classifier design module. In section II, literature review discusses the various approaches done before and proves a solid theoretical basis for this design. Section III introduces the materials used and the functional block diagram in this project. Section IV explains the details of the calculations in feature extraction and the algorithms implemented in classifier design. Section $\mathrm{V}$ analyses the outcomes of the system with the significant advantages listed. Conclusion is given in section VI, which reaffirms the objective of the project with goals achieved and future improvement.

\section{LITERATURE REVIEW}

Emotion classification systems have been developed based on various types of input, for instance speech, facial gestures or physiological measures (e.g. body temperature, pulse) [4]. Though, they are easy to be picked up, their accountability of reflecting human emotion is limited. Since the discovery of EEG signal in 1924, brain signal has been involved in a vast amount of studies, and psychological studies have used EEG signal in numerous research topics for the last few decades. Database for Emotion Analysis using Physiological signals (DEAP) is utilised as the EEG signal resource in that the experiment uses regression for the stimuli, a standard 1020 EEG electrode placement system and a self-assessment manikin's questionnaire to ensure the accuracy of the collected data. There are two models defining emotion states used, Ekman's six universal emotions and Russel's circumplex model. Ekman's model categorises human emotion into six areas, which are anger, disgust, fear, happiness, sadness and surprise [5]. On the other hand, Russel's circumplex model uses a 2D plane to express emotions. The horizontal axis measures the valence, and the vertical axis indicates the arousal scale. Researchers [6][7] have used both models depending on their approaches. In this paper, emotion states are demonstrated in 2-dimension plane with four quadrants.

The second module, feature extraction, defines the types of targets that this project focuses on. It is worth to notice that data segmentation is a necessary step since EEG signal recordings are often lasting a relatively extended period. Henry et al. [8] investigate how different window sizes affect the accuracy. The sufficient window size for arousal and valence is between 3 to 10 and 3 to 12 seconds respectively. Kalaivani et al. [9] consider seven elements from the time 
domain, including amplitude (maximum, minimum and peak to peak) mean, median, standard deviation, variance, skewness, and kurtosis. Using time domain only, the classification accuracy is at approximately 50\%. More researchers argue that much more information hides in the frequency domain. A number of projects [10][11][12][13] use Fourier analysis to generate features. The more advanced method, wavelet transform, can locate every change through the EEG signal. Rosso et al. [14] argue that wavelet energy is capable of detecting variations in a nonstationary signal. As well, it has shorter computational time and the ability to eliminate noises. The accuracy of using frequency domain features is at least $10 \%$ to $20 \%$ higher than the time domain. Therefore, this paper adopts 6 -second time window with wavelet transform analysis to establish the feature variables.

In the last module, classifier design, more than ten different basic algorithms have been studied and modified by researchers seeking for the highest accuracy based on their data and feature variables. Linear Discriminant Analysis, Neural Networks and Nearest Neighbour Classifiers have been implemented in brain-computer interfaces projects. Recent developed algorithms such as relevance vector machine also demonstrate high accuracy percentage. Additionally, another commonly used method is SVM. SVM is known to have good generalization properties and to be insensitive to overtraining [15]. Nonetheless, SVMs are large margin classifiers known for their excellent discrimination performance in binary decision problems but they do not incorporate a model of time [16]. The drawback can be avoided by using a combined classifier of SVM and HMM, which is efficient to represent in time. The combined SVM and HMM approach has been studied in facial expression recognition. However, it has not been introduced in EEG-based emotion classification.

\section{MATERIAL AND METHOD OVERVIEW}

In this design, DEAP is selected to be the experimental input dataset. It has considered the factors of non-invasive and high resolution electrode placement with a self-assessment questionnaire to maximise the accuracy of the collected EEG signal and the emotion states recorded. DEAP dataset contains 32 test subjects who watched 40 music video clips while their EEG signal was collected. Each video clip lasts 60 seconds. The dataset is downloaded from the official website, and the data file is translated into '.mat' format.

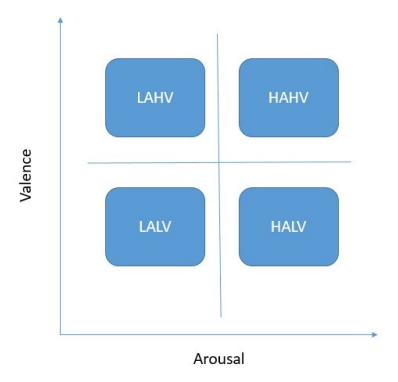

Fig. 1. 2D Emotion Plane
For each subject, there are two arrays that record their EEG signal and corresponding emotion states. The first array includes 40 videos labelled from 1 to 40, 32 EEG electrodes as the Geneva order and 60 seconds EEG signal in millivolts. The other array indicates the scales of the four emotion states while watching the music video clips. For this project, only two variables are chosen, namely valence and arousal levels. The emotion states are measured on the emotion plane as shown in Fig.1. The median value, 5, is used to separate these four areas in both axes.

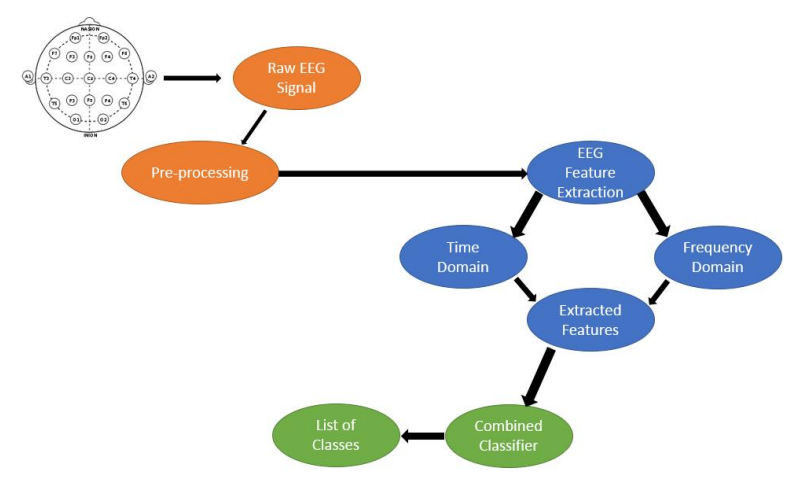

Fig. 2. Functional Block Diagram

The methods overview is demonstrated using a functional block diagram shown in Fig.2. The raw signal is collected from the standard 10-20 system, then pass to pre-processing that removes noises and divides the long signal into 6-second segments. The second module uses wavelet analysis to transfer EEG signal from time domain to frequency domain. Three parameters are calculated from each section forming the extracted features. The features enter the combined SVM and HMM classifier and the output is the classified emotion according to arousal-valence plane.

\section{METHODS}

\section{A. Pre-processing}

The signal is down-sampled to $128 \mathrm{~Hz}$ sample rate. Lower sample rate reduces processing time for the feature parameters calculation. Electrooculography (EOG) is removed in that eye movement is the primary noise source. A bandpass filter is applied to remove noises that are less than $4 \mathrm{~Hz}$ or larger than $45 \mathrm{~Hz}$. The noise-free EEG signal is segmented into 6 second time window. Since the sample rate is $128 \mathrm{~Hz}$, after the segmentation, the total number of segments for this whole project is $10 \times 32 \times 40 \times 32=409600$. All parts will go through the feature extraction process and $30 \%$ of the segments are selected to be the learning set for the classifier, and $70 \%$ are in the testing set.

\section{B. Feature Extraction}

DWT is utilised for this project due to the advantages of time-frequency localisation, multiscale zooming, and noise filtering. Since the project focuses on the valence-arousal plane emotion states, the frequency bands chosen are alpha, beta and gamma bands. The specific variables for wavelet 
transform are determined based on previous studies. The mother wavelet function is Daubechies5 (db5) and the decomposition level is 6 based on the dominant frequency components of EEG signal which are from $8 \mathrm{~Hz}$ to $64 \mathrm{~Hz}$.

To start with wavelet analysis, DWT coefficients are calculated. The following equation finds the wavelet coefficients.

$$
<f, \psi_{a, b}>=C_{a, b}=\int_{-\infty}^{\infty}\left(\frac{1}{\sqrt{2^{-j}}}\right) \psi\left(\frac{t-2^{-j} k}{2^{-j}}\right)
$$

In equation (1), $2^{j} k$ and $2^{j}$ are the time localisation and scale respectively, while $\psi(t)$ denotes the mother wavelet function [8]. DWT coefficients are used in all three features calculation.

The first feature is relative wavelet energy. It is calculated as follows:

$$
p_{j}=\frac{\sum_{k}^{N}\left|C_{j}(k)\right|^{2}}{\sum_{j} \sum_{k}\left|C_{j}(k)\right|^{2}}
$$

$C_{j}(k)$ represents the detail coefficients, which indicates that the numerator is the detail wavelet energy. The denominator is the total wavelet energy. The probability $p_{j}$ reveals the time-scale density of the input data.

The second feature extracted is relative wavelet entropy. It is expressed below:

$$
S_{w t}(p \mid q)=\sum_{j} p_{j} \cdot \ln \left(\frac{p_{j}}{q_{j}}\right)
$$

The variable $q_{j}$ is used as the reference distribution to give a more accurate value for $p_{j}$. Relative wavelet entropy shows the similarity between two probabilities. In this design, the Shannon entropy is utilised.

The third feature used in the project is an innovative approach of joining two features together. DWT coefficients and standard deviation are selected and multiplied to create this variable. This method proposes an ideal circumstance that it includes information from both time domain and frequency domain, which leads to better performance of the classification system. The mathematical notation is shown in equation (4).

$$
\lambda=C_{a, b} \cdot \sigma^{2}
$$

\section{Classifier Design}

Firstly, SVM is developed to classify the extracted features. Since SVM is normally for binary classification, in order to classify both valence and arousal axes, two SVMs are established. Radial Basis Function is utilised in the training step. There are three combinations tested in SVM classifier: the innovative variable that joins both time and frequency domain; a bundle of two variables from DWT; and a bundle of all three variables.

While SVMs are large-margin classifiers known for their excellent discrimination performance in binary decision problems, they do not incorporate a model with time, HMM is capable of representing temporal dynamics very efficiently.
Thus, a combined model of both SVM and HMM is designed to reach higher accuracy. The general idea is to use SVM output as the input of HMM. However, it is not possible to directly use the output as a probability measure. The output indicates the distance between the sample to the support vectors and the relationship between the output and the class probability is not clear. A sigmoid function is utilised to discover the connection between these two variables shown below:

$$
g(h(x), A, B)=\frac{1}{1+\exp (A h(x)+B)}
$$

The parameters $\mathrm{A}$ and $\mathrm{B}$ are calculated by maximum likelihood estimation with $g(h(x), A, B)$. The distance between the support vectors and the optimised plane is used to create the HMM classifier model. Since the classifier only needs to identify whether the input signal is on the higher half or the lower half of the axes, using MATLAB HMM toolbox function, hmmtrain and hmmdecode, the class probability is successfully reflected.

\section{RESULTS AND DISCUSSION}

TABLE I

AVERAGE ACCURACY FOR DIFFERENT FEATURE COMBINATIONS

\begin{tabular}{|c|c|c|c|}
\hline $\begin{array}{c}\text { 2D } \\
\text { Plane }\end{array}$ & $\begin{array}{c}\text { SVM (Innovative } \\
\text { Variable Only) }\end{array}$ & $\begin{array}{c}\text { SVM (Wavelet } \\
\text { Variables) }\end{array}$ & $\begin{array}{c}\text { SVM (All Three } \\
\text { Variables) }\end{array}$ \\
\hline Valence & 0.5793 & 0.5485 & 0.6021 \\
\hline Arousal & 0.6136 & 0.6182 & 0.6279 \\
\hline
\end{tabular}

The contribution of the innovative variable for emotion classification is studied. SVM classifier is used with three combinations of the extracted feature shown in Table I. The first set uses the innovative variable only. The second set contains both of the wavelet analysis variables. The last set is a three dimensional matrix including all of the three features. It is worth to note that all 40 music clips and 32 test subjects are taken into the calculation. Since it is a large dataset, average accuracy on valence and arousal planes are used to represent the overall performance. Despite the accuracy of individual parameters is relatively low; the joined three parameter data group is consistently higher than other combinations. On valence axis, the increase of the accuracy of the three parameters test is more than $2 \%$ for innovative parameter only and more than $5 \%$ for wavelet parameters only. The increase of accuracy confirms that combining time and frequency domain parameters can improve the performance of the classification system.

The SVM classifier and the combined classifier are tested seperately by the three dimentional dataset shown in the last column of Table I. Fig.3 and Fig.4 demonstrate the average accuracy for each test subject on both valence and arousal plane. As the diagrams shown, the combined SVM and HMM classifier returns higher values constantly in the majority of the cases. This reaffirms the combined classifier methodology, which is to use the property of the support 


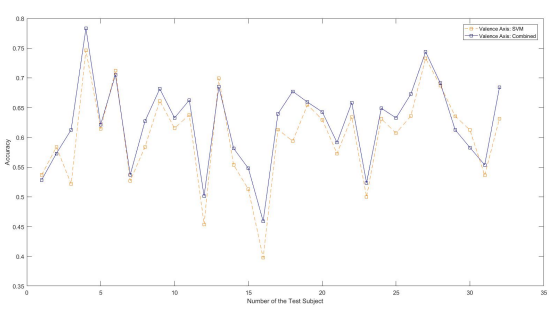

Fig. 3. Valence Plane Average Accuracy for 32 Test Subjects

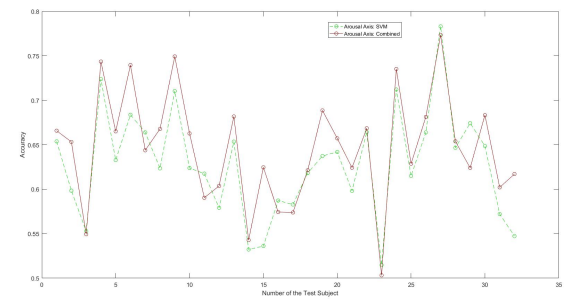

Fig. 4. Arousal Plane Average Accuracy for 32 Test Subjects

vector of the SVM classifier as the input of the HMM classifier, and as a result, the second classifier finds the hidden information in the features and corrects the classification process.

Fig.5 compares the average accuracy for these two classifiers on valence and arousal axis. The blue bars are the average accuracy for SVM classifier and the yellow bars stand for the combined design. The average accuracy on valence axis rises $3.15 \%$ and it increases $2.88 \%$ on arousal axis. The stable increase of accuracy on both axes illustrates that the combined SVM and HMM classifier contributes in improving the accuracy and the stability of the system.

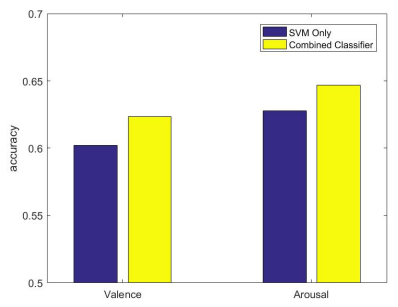

Fig. 5. Average Accuracy: SVM vs Combined Classifier

\section{CONCLUSIONS}

This paper establishes an innovative design of emotion classification system based on EEG signal. Using DEAP database as the input data, three features, relative wavelet energy, relative wavelet entropy and a novel variable which is the product of standard deviations in time domain and DWT coefficients from frequency domain are calculated respectively. A combined SVM and HMM classifier is also designed aiming to improve the accuracy of the classification. The result indicates that the bundle containing all three features returns the highest accuracy. Comparing the combined classifier with solo SVM classifier, the accuracy increases approximate $3 \%$. It indicates that the combined classifier can optimise the emotion recognition process consistently.

The future improvement includes that the SVM classifier could be tuned to return a higher accuracy using customise Kernel function. As well, other algorithm combinations could be tested.

\section{REFERENCES}

[1] J. D. Mayer and G. Geher, "Emotional intelligence and the identification of emotion," Intelligence, vol. 22, no. 2, pp. 89-113, 1996

[2] X.-W. Wang, D. Nie, and B.-L. Lu, "Emotional state classification from EEG data using machine learning approach," Neurocomputing, vol. 129, pp. 94-106, 2014.

[3] L. Xin, S. Xiao-Qi, Q. Xiao-Ying, and S. Xiao-Feng, "Relevance Vector Machine Based EEG Emotion Recognition," in Instrumentation \& Measurement, Computer, Communication and Control (IMCCC), 2016 Sixth International Conference on, 2016, pp. 293-297: IEEE

[4] P. Ackermann, C. Kohlschein, J. . Bitsch, K. Wehrle, and S. Jeschke, "EEG-based automatic emotion recognition: Feature extraction, selection and classification methods," in e-Health Networking, Applications and Services (Healthcom), 2016 IEEE 18th International Conference on, 2016, pp. 1-6: IEEE.

[5] R. W. Levenson, L. L. Carstensen, W. V. Friesen, and P. Ekman, "Emotion, physiology, and expression in old age," Psychology and aging, vol. 6, no. 1, p. 28, 1991.

[6] A. Bhardwaj, A. Gupta, P. Jain, A. Rani, and J. Yadav, "Classification of human emotions from EEG signals using SVM and LDA Classifiers," in Signal Processing and Integrated Networks (SPIN), 2015 2nd International Conference on, 2015, pp. 180-185: IEEE.

[7] Y.-H. Liu, C.-T. Wu, Y.-H. Kao, and Y.-T. Chen, ”Single-trial EEGbased emotion recognition using kernel Eigen-emotion pattern and adaptive support vector machine," in Engineering in Medicine and Biology Society (EMBC), 2013 35th Annual International Conference of the IEEE, 2013, pp. 4306-4309: IEEE.

[8] $\mathrm{H}$. Candra et al., "Investigation of window size in classification of EEG-emotion signal with wavelet entropy and support vector machine," in Engineering in Medicine and Biology Society (EMBC), 2015 37th Annual International Conference of the IEEE, 2015, pp. 7250-7253: IEEE.

[9] M. Kalaivani, V. Kalaivani, and V. A. Devi, "Analysis of EEG Signal for the Detection of Brain Abnormalities," at International Journal of Computer Applications year, 2014.

[10] Y. Yuankui and Z. Jianzhong, "Recognition and analyses of EEG \& ERP signals related to emotion: from the perspective of psychology," in Neural Interface and Control, 2005. Proceedings. 2005 First International Conference on, 2005, pp. 96-99: IEE

[11] R.-N. Duan, J.-Y. Zhu, and B.-L. Lu, ”Differential entropy feature for EEG-based emotion classification," in Neural Engineering (NER), 2013 6th International IEEE/EMBS Conference on, 2013, pp. 81-84: IEEE.

[12] Y.-P. Lin et al., "EEG-based emotion recognition in music listening," Biomedical Engineering, IEEE Transactions on, vol. 57, no. 7, pp. 1798-1806, 2010.

[13] R. M. Ravindran, "Classification of human emotions from EEG signals using filtering and ANFIS classifier," in Current Trends in Engineering and Technology (ICCTET), 2014 2nd International Conference on, 2014, pp. 113-119: IEEE.

[14] O. A. Rosso et al., "Wavelet entropy: a new tool for analysis of short duration brain electrical signals," Journal of neuroscience methods, vol. 105, no. 1, pp. 65-75, 2001.

[15] F. Lotte, M. Congedo, A. Lcuyer, F. Lamarche, and B. Arnaldi, "A review of classification algorithms for EEG-based brain?computer interfaces," Journal of neural engineering, vol. 4, no. 2, p. R1, 2007.

[16] M. F. Valstar and M. Pantic, "Combined support vector machines and hidden markov models for modeling facial action temporal dynamics," in International Workshop on Human-Computer Interaction, 2007, pp. 118-127: Springer. 